\title{
Phagocytosis of neonatal pathogens by peripheral blood neutrophils and monocytes from newborn preterm and term infants
}

\author{
Amy Prosser ${ }^{1}$, Julie Hibbert ${ }^{1}$, Tobias Strunk ${ }^{1,2}$, Chooi Heen Kok ${ }^{2}$, Karen Simmer ${ }^{2}$, Peter Richmond ${ }^{1}$, David Burgner ${ }^{1,3,4}$ \\ and Andrew Currie 2,5
}

BACKGROUND: Deficiencies in phagocytosis may contribute to the increased susceptibility of infants to early life infections. Data on phagocytosis of the major neonatal pathogens Staphylococcus epidermidis (SE), Staphylococcus aureus (SA), and Escherichia coli (EC) by preterm infant leukocytes are inconsistent.

METHODS: Cord and <24-h peripheral blood were collected from very preterm (<30.1 wks gestational age (GA)) and term (37-42 wks GA) infants. Monocyte and neutrophil phagocytosis of pHrodo-labeled SE, SA, and EC were analyzed using a small-volume flow cytometry assay, with simultaneous characterization of surface activation marker expression.

RESULTS: Preterm infants had lower proportions of monocytes and neutrophils capable of phagocytosis than term infants, but preterm infant phagocytes had higher phagocytic capacity. Phagocytosis was strongly correlated between cord and $<24-h$ peripheral blood. Supplementation with exogenous complement significantly increased phagocytosis of EC but not of SE or SA. Monocyte human leukocyte antigen (HLA)-DR expression was lower in preterm infants but did not correlate with phagocytosis.

CONCLUSION: There is no defect in phagocytosis by monocytes and neutrophils from preterm compared with term infants, although preterm infants possess fewer phagocytes, possibly contributing to susceptibility to bacterial infection. Further investigation into the development of postnatal phagocytic competence is warranted.

P hagocytosis is an essential component of innate immune responses to pathogens, encompassing recognition, binding, engulfment, and degradation of particles such as bacteria. Although primarily an innate immune process, degradation and processing of microbes during phagocytosis for presentation via major histocompatibility complexes is also a crucial link to adaptive immune system responses (1). Individuals with phagocytic defects (e.g., Wiskott-Aldrich syndrome (2)) are at an increased risk of bacterial infection.
Very preterm infants ( $<30$ wks gestational age (GA)) are extremely susceptible to bacterial infection, with risk inversely correlated with birth weight and GA $(3,4)$. Earlyand late-onset sepsis (occurring before and after $72 \mathrm{~h}$, respectively) remain important causes of morbidity and mortality $(4,5)$. The commonest pathogen group in preterm infants is coagulase-negative staphylococci, of which Staphylococcus epidermidis (SE) is the predominant species. Coagulase-negative staphylococci are responsible for $\sim 50 \%$ of late-onset sepsis and 7-12\% of early-onset sepsis episodes (4-6). Escherichia coli (EC), Staphylococcus aureus (SA) and Group B Streptococcus (GBS) account for the majority of non-coagulase-negative staphylococci infections in preterm infants $(5,7,8)$.

Deficiencies of innate immune functions, including phagocytosis, cytokine production, and complement activity, have been reported in preterm infants and are suggested to contribute to their heightened susceptibility to bacterial infection $(9,10)$. However, the data, particularly for deficiencies in phagocytosis, are inconsistent (11). There are no definitive comparisons of phagocytosis between adults and neonates; studies have identified positive $(12,13)$, negative $(14)$, and neutral $(11,13)$ trends in functionality between the groups $(15)$. Similarly, the reported correlation between GA and phagocytosis functionality is inconsistent $(12,16)$. Discrepancies are also evident when comparing specific cell types, including monocytes and neutrophils (12-15).

Characterization of innate immune responses to common neonatal pathogens is essential to inform translational studies aiming to reduce the disease burden. However, immune studies of preterm cells is challenging, as blood samples from preterm infants (with a blood volume of $<50 \mathrm{ml}$ (17)) are limited in volume. Previous studies of preterm phagocytic responses to bacterial infection have largely used cord blood, as comparatively large volumes $(5-30 \mathrm{ml})$ are readily obtained. However, characterization of postnatal peripheral blood is essential; there are few data on changes in innate

\footnotetext{
'School of Paediatrics and Child Health, University of Western Australia, Perth, Western Australia, Australia; ${ }^{2}$ University of Western Australia Centre for Neonatal Research and Education, Perth, Western Australia, Australia; ${ }^{3}$ Murdoch Childrens Research Institute, Royal Children's Hospital, Parkville, Victoria, Australia; ${ }^{4}$ Department of Paediatrics, University of Melbourne, Parkville, Victoria, Australia; ${ }^{5}$ School of Veterinary \& Life Sciences, Murdoch University, Perth, Western Australia, Australia. Correspondence: Andrew Currie (A.Currie@murdoch.edu.au) 


\section{Articles | Prosseretal.}

immune function in the immediate postnatal period and it is unclear whether cord blood data are representative of peripheral blood responses.

To enable analysis of very preterm peripheral blood, we developed and optimized a small-volume, whole-blood assay utilizing a $\mathrm{pH}$-sensitive dye pHrodo to detect monocyte and neutrophil phagocytic functionality. The formation of the phagosome around an ingested entity during phagocytosis relies on low $\mathrm{pH}$ for bactericidal functions and antigen processing and presentation $(1,18)$. pHrodo is a highly $\mathrm{pH}$-sensitive succinimidyl ester dye that fluoresces dramatically when exposed to low $\mathrm{pH}(<4.0)$. Phagocytosis is therefore detectable and quantifiable when the phagosome forms, and acidifies, around ingested pHrodo-labeled bacteria, minimizing high background fluorescence of cell-bound, but uningested, bacteria $(18,19)$.

Our aims are to (i) describe this novel technique, (ii) compare monocyte and neutrophil phagocytic capacity in cord and peripheral blood, (iii) examine the effect of GA on phagocytosis by monocytes and neutrophils of common neonatal pathogens SE, SA, and EC, and (iv) investigate correlations between cellular activation marker expression and phagocytosis activity.
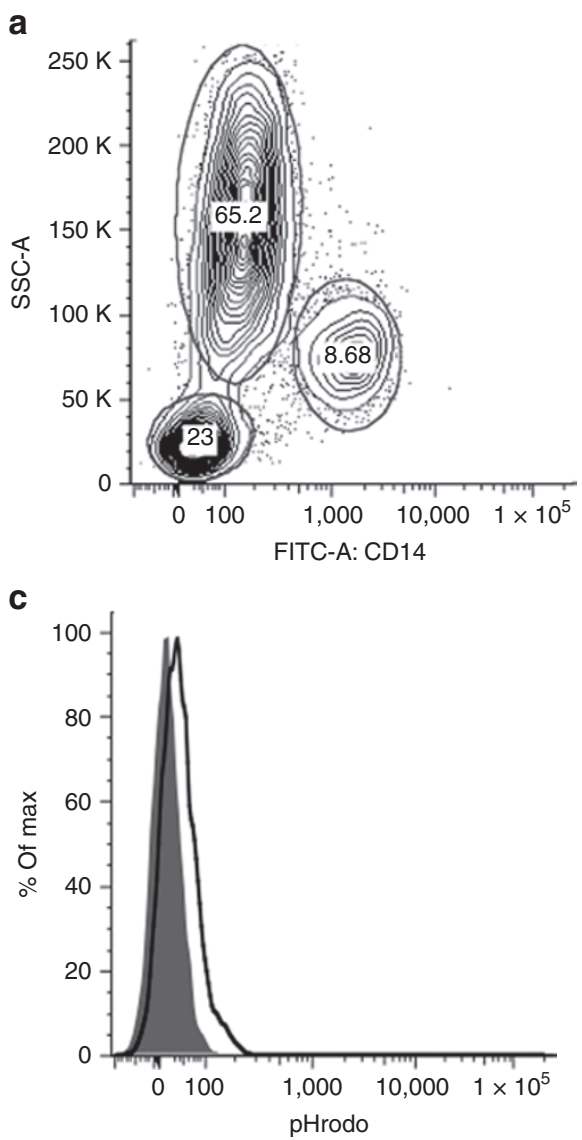

\section{RESULTS}

Detection of Phagocytosis With pHrodo-Labeled Bacteria Using Whole Blood

The phagocytosis assay conditions used for neonatal samples were initially optimized for time, dose, and minimum blood volume requirement using peripheral blood from healthy adult volunteers (data not shown). Incubation of preterm infant whole blood with pHrodo-labeled bacteria, under optimal conditions, resulted in a marked shift in fluorescence of phagocytic cells clearly detectable above the background of unstimulated blood (Figure 1b,1d). Both phagocyte populations (monocytes and neutrophils) were readily identified in preterm infant samples using a combination of anti-CD14 staining and side-scatter properties (Figure 1a). The percentage of pHrodo $^{+}$cells and the associated median pHrodo fluorescence intensity (MFI) of phagocytic cells were readily determined. As expected, nonphagocytic lymphocytes did not fluoresce (Figure 1c). Blocking of phagocytosis in adult blood by preincubation with $20 \mu \mathrm{g} / \mathrm{ml}$ cytochalasin $\mathrm{D}(\mathrm{CD})$ was observed although complete abrogation of phagocytosis was not achieved (data not shown). Confocal microscopy of adult blood with pHrodo-labeled SE, with or without the addition of CD showed low levels of fluorescence of bacteria associated
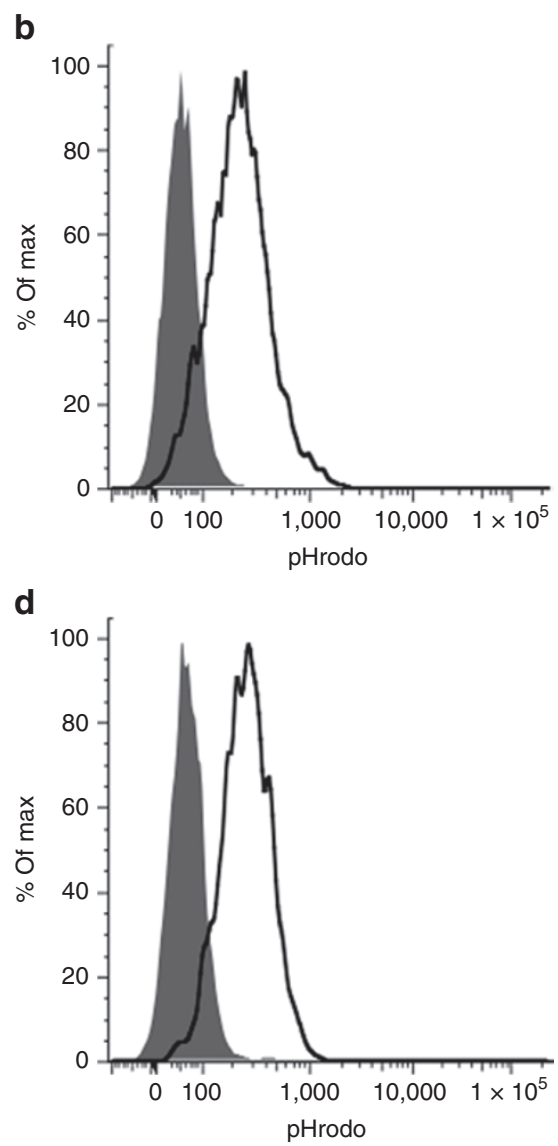

Figure 1. Analysis of phagocytosis using pHrodo-labeled bacteria. (a) Representative plot of an infant peripheral blood sample collected under the optimized phagocytosis protocol, showing inclusion gates and percentages for monocytes, neutrophils, and lymphocytes based on side-scatter area (SSC-A) and CD14 staining. (b-d) Representative histograms from (a) showing pHrodo fluorescence in the presence (solid black line) or absence (shaded grey) of pHrodo-labeled SE by neutrophils, lymphocytes, or monocytes, respectively. 
with CD-treated cells in comparison with bright fluorescence following phagocytic engulfment (data not shown). Assay optimization was conducted using in-house pHrodo-labeled $\mathrm{SE}$; however, conditions were maintained for commercially purchased pHrodo-labeled SA and EC to ensure comparability during analysis.

Phagocytosis of Important Neonatal Pathogens by Infant Peripheral Blood

Phagocytosis of SE, SA, and EC by $<24$ h peripheral blood cells was assessed and compared between preterm and term infants (Figure 2). Monocytes and neutrophils from all infants were able to phagocytose all three bacterial species, although SE was preferentially phagocytosed over SA and EC. Term infant blood samples had a significantly higher proportion of both monocytes and neutrophils capable of SE and EC ingestion than preterm infants (SE: 66 vs. $45 \%, P=0.0235$; and 54 vs. $36 \%, P=0.0207$, EC: 15 vs. $6 \%, P=0.0001$; and 12 vs. $3 \%, P<$ 0.0001 for monocytes and neutrophils, respectively). However, the level of SA and EC uptake (MFI) by preterm infant monocytes and neutrophils capable of phagocytosis was significantly higher than that by term infants (SA: 222 vs. $184, P=0.0013$; and 204 vs. $160, P=0.0002$, EC: 208 vs. $172, P=0.0104$; and 171 vs. $140, P=0.0003$ for monocytes and neutrophils, respectively). Similar trends were observed for SE (314 vs. 259, $P=$ 0.5861 and 212 vs. $179, P=0.0462$, for monocytes and neutrophils, respectively; Figure 2).

Cord Blood Phagocytosis Responses Are a Good Surrogate for $<24 \mathrm{~h}$ Peripheral Blood Responses

While examination of peripheral blood is ideal for determining postnatal phagocytic responses, cord blood is routinely used as a surrogate for many preterm infant studies. We therefore investigated whether cord blood phagocytic responses are representative of peripheral blood responses of 1-day-old neonates. The proportion of phagocytic monocytes and neutrophils in cord and $<24 \mathrm{~h}$ infant blood were significantly correlated $(P<0.0001$ using nonparametric Spearman correlation) directly and strongly for all bacteria tested (SE: $r=0.76$ and 0.83 ; SA: $r=$ 0.68 and 0.76 ; and EC: $r=0.70$ and 0.72 for monocytes and neutrophils, respectively; Figure 3). The degree of bacterial uptake (MFI) by monocytes and neutrophils also correlated between cord and $<24 \mathrm{~h}$ samples for all bacterial species, although less strongly than for the percentage of positive cells (SE: $r=0.77$ and 0.76; SA: $r=0.67$ and 0.78; and EC: $r=0.58$ and 0.70 for monocytes and neutrophils, respectively; $P<0.0001$ all comparisons, data not shown).

Cellular Activation Status Does Not Correlate With the Levels of Phagocytosis in Preterm and Term Infants

Expression levels of activation markers on phagocytes were determined to establish if the phagocytic differences observed between preterm and term infants were due to differences in cellular activation. Monocyte expression of human leukocyte antigen (HLA)-DR and CD86, and neutrophil expression of CD64, were compared between preterm and term infants using $<24$ h blood samples. Monocyte expression of CD86 was comparable between preterm and term infants, as was neutrophil expression of CD64, with no significant difference between the groups for either marker. Of note, expression of the monocyte lineage and activation marker HLA-DR was more than twofold higher on monocytes from term compared with preterm infants (MFI of 5,584 vs. 2,500, $P=0.0003$; Figure 4). The effect of differential HLA-DR expression was investigated by
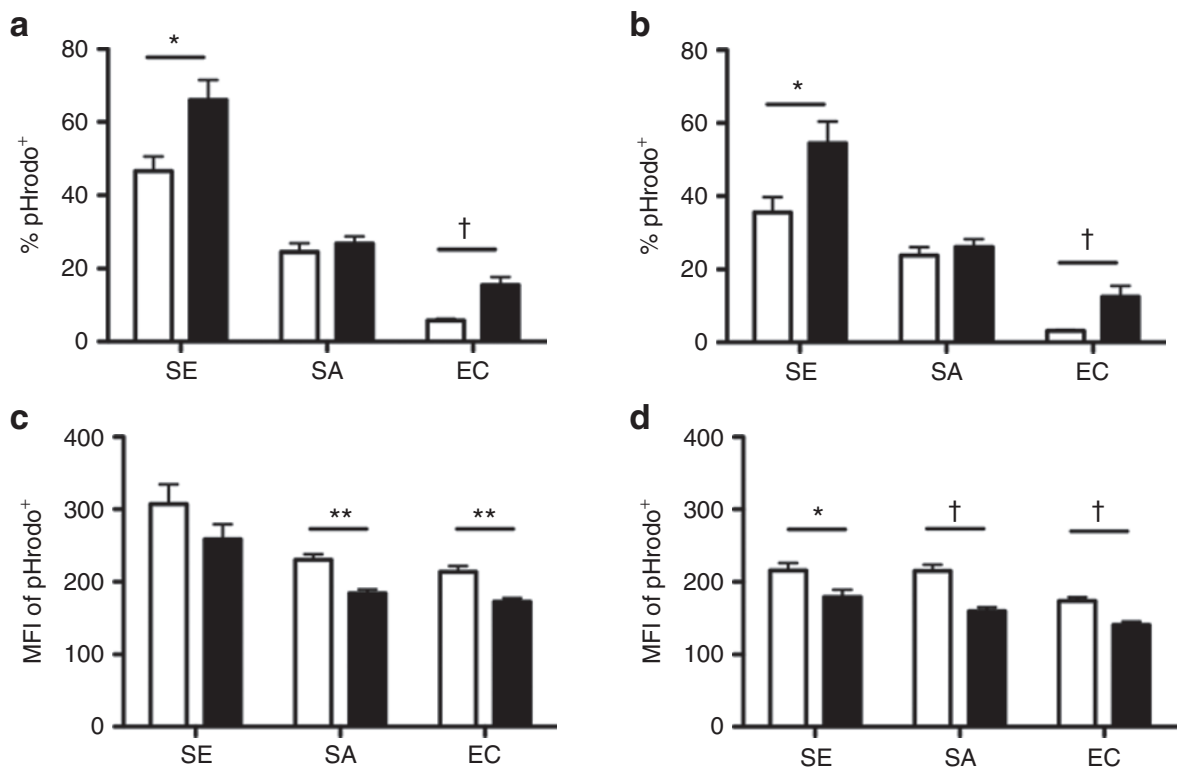

Figure 2. Phagocytosis of $\mathrm{SE}, \mathrm{SA}$, and $\mathrm{EC}$ by term and preterm infants. Peripheral $(<24 \mathrm{~h}$ postnatal) blood samples from very preterm $(<30.1$ wks $\mathrm{GA}$, white bars, $n=57-58$ ) and term (37-42 wks GA, black bars, $n=20)$ infants were incubated with $1 \times 10^{8}$ bacteria/ml before analysis by flow cytometry. The percentages of pHrodo ${ }^{+}$monocytes (a) and neutrophils (b) and MFI of pHrodo + monocytes (c) and neutrophils (d) were compared. ${ }^{*} P<0.05$; ${ }^{*} P<0.005 ;{ }^{+} P<0.0001$. EC, Escherichia coli; MFI, median pHrodo fluorescence intensity; SA, Staphylococcus aureus; SE, Staphylococcus epidermidis. 


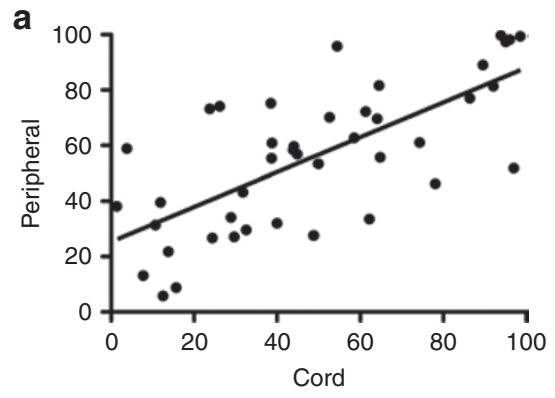

d

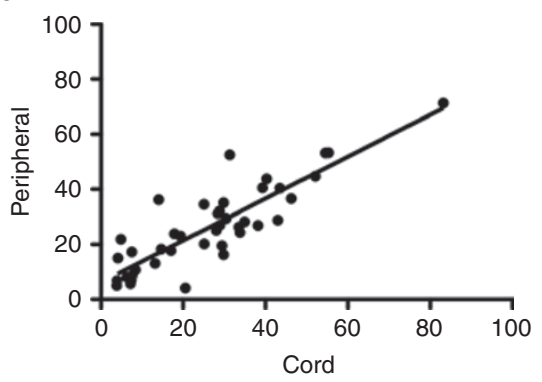

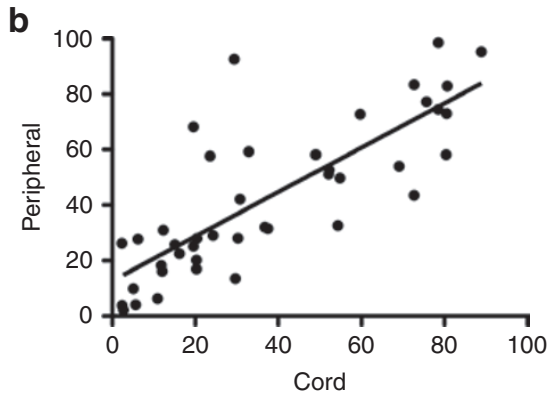

e

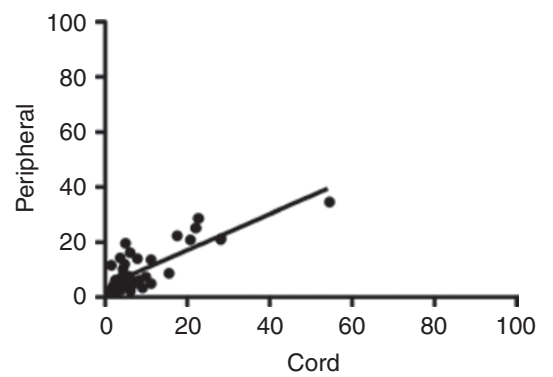

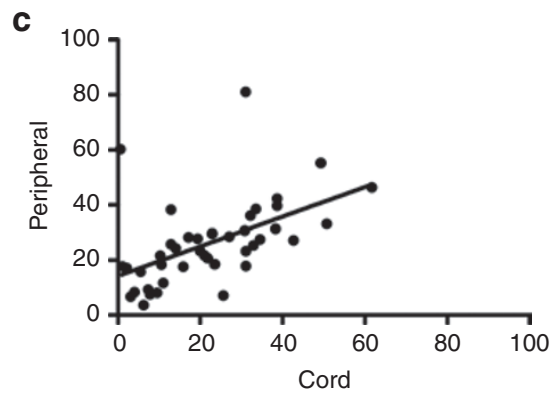

f

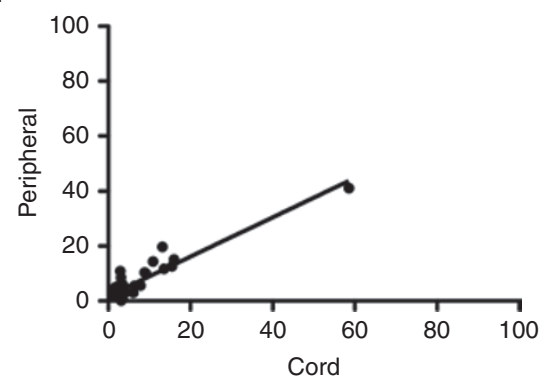

Figure 3. Correlation of infant ( $<30-42$ wks GA) cord and peripheral blood phagocytosis of SE $(n=42)$, SA $(n=41)$ and EC ( $n=42)$ by monocytes (panels $\mathbf{a}, \mathbf{c}$, and $\mathbf{e}$, respectively) and neutrophils (b, $\mathbf{d}$, and $\mathbf{f}$, respectively). Data shown are percentages of pHrodo ${ }^{+}$phagocytes. The degree of correlation was determined using the nonparametric Spearman correlation with $r$ values as follows: $\mathbf{a}=0.76, \mathbf{b}=0.83, \mathbf{c}: r=0.68, \mathbf{d}: r=0.76, \mathbf{e}: r=0.70, \mathbf{f}: r=0.72$. $P<$ 0.0001 for all correlations. EC, Escherichia coli; SA, Staphylococcus aureus; SE, Staphylococcus epidermidis.

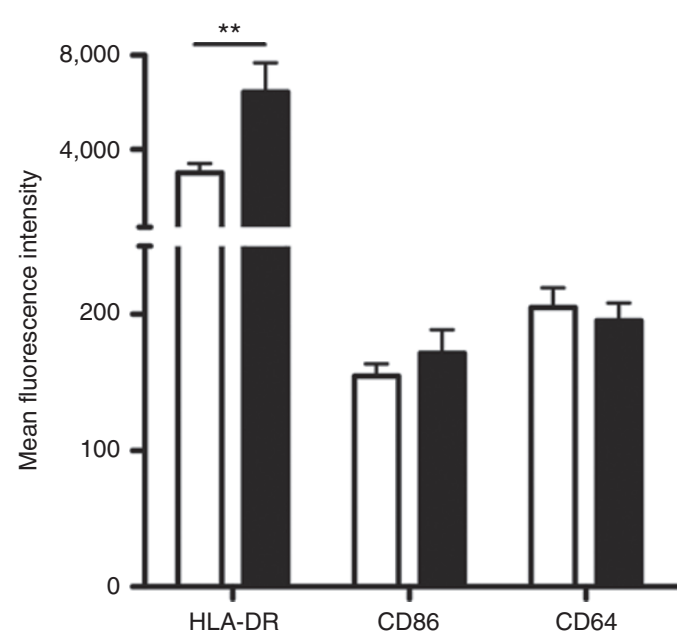

Figure 4. Expression levels of monocyte and neutrophil activation markers. Peripheral blood from preterm ( $n=57$; white bars) and term ( $n=19$; black bars) infants was incubated with anti-HLA-DR, anti-CD86, and anti-CD64 antibodies before analysis by flow cytometry. Data plotted are the median fluorescence intensity (MFI) of HLA-DR and CD86 expression by monocytes (identified as Lin-2 $2^{+} \mathrm{HLA}^{-D R^{+}}$) and CD64 expression by neutrophils (identified as SSChiLin2-). ${ }^{*} P=0.0003$. HLA, human leukocyte antigen.

correlating expression levels with phagocytosis (both proportion of phagocytic cells and MFI). No significant correlation of either measure of phagocytosis with HLA-DR expression was observed (data not shown).

\section{Addition of Exogenous RbC Selectively Enhances Phagocytosis in Preterm Infants}

Hypocomplementemia is suggested to be critical in preterm infant phagocytic function (20). We therefore investigated the role of complement by adding whole blood to pHrodo-labeled bacteria with or without rabbit complement $(\mathrm{RbC})$ and comparing the nonopsonic phagocytic capability of each sample and the potentially additive effect of exogenous complement. The proportion of cells phagocytosing EC was significantly increased in both term and preterm neutrophils and preterm monocytes, whereas phagocytosis of SE and SA was not altered for either cell type with the addition of RbC (Table 1).

\section{DISCUSSION}

The simultaneous assessment of multiple bacterial species and cell types and the various outputs for phagocytosis described is the first definitive description of the differences in preterm and term infant phagocytic capabilities. Preterm infants have fewer peripheral blood monocytes and neutrophils capable of bacterial phagocytosis than term infants, but their remaining phagocytes possess higher capacity for bacterial uptake than those from term infants. These differences were independent of phagocyte activation and, for SE and SA, not influenced by exogenous complement. We also demonstrate that cord blood provides a reasonable surrogate for $<24 \mathrm{~h}$ peripheral blood from preterm and term infants.

The development of an assay to simultaneously detect phagocytosis by monocytes and neutrophils in a minimal whole blood volume was essential. This is a broadly relevant issue as current data on neonatal phagocytosis, largely derived from cord blood are conflicting and their relevance to the age at which neonatal sepsis occurs in vivo is unclear. Previous studies have reported both positive (16) and negative (12) correlations between GA and phagocytosis. Furthermore, studies comparing phagocytosis activity of monocytes and neutrophils from adults and 
Table 1. Phagocytosis with and without exogenous rabbit complement (RbC)

\begin{tabular}{|c|c|c|c|c|c|c|c|c|}
\hline \multirow[b]{2}{*}{$\%$} & \multicolumn{4}{|c|}{ Monocytes } & \multicolumn{4}{|c|}{ Neutrophils } \\
\hline & WB & $+\mathrm{RbC}$ & WB & $+\mathrm{RbC}$ & WB & $+\mathrm{RbC}$ & WB & $+\mathrm{RbC}$ \\
\hline SE & $46 \pm 5$ & $40 \pm 5$ & $66 \pm 5$ & $53 \pm 6$ & $36 \pm 5$ & $33 \pm 5$ & $54 \pm 6$ & $46 \pm 5$ \\
\hline SA & $24 \pm 3$ & $25 \pm 3$ & $27 \pm 2$ & $24 \pm 2$ & $22 \pm 2$ & $27 \pm 3$ & $26 \pm 2$ & $28 \pm 3$ \\
\hline EC & $6 \pm 1$ & $11 \pm 1^{\mathrm{a}}$ & $16 \pm 2$ & $15 \pm 2$ & $3 \pm 1$ & $12 \pm 2^{b}$ & $12 \pm 3$ & $18 \pm 2^{c}$ \\
\hline SE & $314 \pm 39$ & $275 \pm 27$ & $259 \pm 20$ & $220 \pm 12$ & $212 \pm 12$ & $207 \pm 10$ & $179 \pm 10$ & $169 \pm 8$ \\
\hline SA & $223 \pm 9$ & $224 \pm 10$ & $184 \pm 5$ & $184 \pm 6$ & $205 \pm 9$ & $211 \pm 10$ & $160 \pm 5$ & $165 \pm 7$ \\
\hline EC & $208 \pm 9$ & $204 \pm 11$ & $173 \pm 5$ & $169 \pm 5$ & $171 \pm 7$ & $181 \pm 9$ & $141 \pm 5$ & $145 \pm 4$ \\
\hline \multicolumn{9}{|c|}{ EC, Escherichia coli; MFI, median pHrodo fluorescence intensity; SA, Staphylococcus aureus; SE, Staphylococcus epidermidis; WB, whole blood. } \\
\hline
\end{tabular}

neonates have reported diminished (14), enhanced $(12,13)$, or equivalent capability $(11,13)$. These inconsistent findings are likely to reflect methodological differences, as sample type (e.g., purified cells or whole blood), preparations and species of bacteria, together with the use of humoral factors (e.g., complement) vary considerably between studies (11). Although previous studies have utilized pHrodo as a tool for assessing phagocytosis, none have extended its use to simultaneous analysis of multiple cell types, or to whole blood assays $(18,21)$.

We found that preterm infants possess a decreased proportion of whole-blood phagocytes capable of phagocytosing the common neonatal pathogens SE, EC and, to a lesser extent, SA compared with term infants. The paucity of current studies comparing phagocytosis functionality between term and preterm infants and the variations in methodologies employed make it difficult to compare our data to published observations. However, the decreases observed in the proportion of phagocytic cells in preterm compared with term infants are supported by previous investigations of neutrophil phagocytosis of EC and SA $(22,23)$. The higher phagocytic potential in preterm infants observed in our study has also been previously reported for phagocytosis of EC by monocytes and GBS by neutrophils $(12,24)$. By contrast, other studies and our own previous data have described reduced or equivalent phagocytic potential of neutrophils and monocytes from preterm and term infants for EC, SE, or GBS $(22,24,25)$. These discrepant data are most likely due to methodological differences in assessing phagocytosis.

The key observation of preferential phagocytosis of bacteria, particularly SE, by monocytes compared with neutrophils has not been previously reported. These findings, if corroborated, may guide future interventions that target specific cell types that are most important in phagocytosing specific pathogens. In keeping with this approach, we have previously shown that monocytes are the key cytokine-producing cell type when exposed to SE or GBS and that phagocytosis is essential for stimulating cytokine production in these cells (24).
The phagocytic responses of neutrophils and monocytes from cord and $<24 \mathrm{~h}$ peripheral samples were strongly correlated and highly comparable, indicating that phagocytosis functionality is maintained over this relatively short yet clinically relevant time frame.

Cord blood is routinely used as a noninvasive, easily accessible neonatal sample as a surrogate for peripheral samples in immunological studies $(24,26,27)$. However, the correlation between phagocytosis in cord and peripheral blood has not been reported previously. Phagocytosis of EC by monocytes from cord blood and day 3 peripheral blood has been investigated but no statistical comparisons were reported (28). Our data are therefore important in verifying cord blood as a suitable and convenient surrogate to explore early peripheral responses in infants. Our optimized, low sample volume protocol allows investigation of phagocytosis of peripheral samples in the first weeks of life, the time of greatest risk for early-onset sepsis and late-onset sepsis (3).

Variations in phagocytosis levels in response to different bacteria were as anticipated. Many bacteria have developed immune evasion mechanisms, such as polysaccharide capsules, surface expression of protein A causing incorrect orientation of opsonizing antibodies and the antiphagocytic effect of lipopolysaccharide (29-31). These different mechanisms may account for the differential phagocytosis of SE, SA, and EC, indicating pathogen-specific phagocytic capabilities of preterm and term infants. Neutrophils have previously been shown to phagocytose unopsonized SE more readily than EC, as we observed (32). The more effective phagocytosis of SE over SA and EC, although counter-intuitive, suggests that there is no defect in preterm phagocytosis of the most common pathogen in this population. Instead, the pathogenicity of SE appears to be reliant on evasion of the postengulfment killing mechanism of phagocytes. One possible caveat to these findings is the necessary use of heat-killed bacterial preparations, and commercially prepared SA and EC stocks that are non-neonatal sepsis isolates, which may influence interactions 
with infant phagocytes. We have previously shown that heatkilled SE activates a reduced set of innate immune responses in comparison to live SE, at least in terms of cytokine release (33). Future studies could examine the real-time phagocytosis of live, neonatal bacterial isolates by infant monocytes and neutrophils (18). However, this would first require modification and optimization of the methodologies used, in order to ensure they are compatible with the very small postnatal blood volumes available from extremely preterm infants.

The activation markers HLA-DR, CD64, and CD86 have previously been associated with early diagnosis and/or prognosis of sepsis and bacterial infection (34-36). Although this is the focus of much of the investigation into HLA-DR expression, this is the first study to correlate expression with phagocytosis. Of note, although expression levels were significantly lower in preterm infants, as previously described $(36,37)$, there was no correlation of expression with reduced phagocytosis, suggesting that the prognostic utility of HLA-DR expression is independent of phagocytosis functionality. The difference in HLA-DR expression and any effect on phagocytosis may be transient, as others have reported equivalent expression levels between preterm and term infants up to 7-9 d of age (38). Similarly, expression of CD64 on neutrophils and CD86 on monocytes was not correlated with phagocytic activity. Levels were comparable between preterm and term infants, as previously described for CD64 $(35,39)$, although decreased CD86 has been reported in preterm neonates (37). These results are perhaps not surprising, given that all preterm infants included in this study were healthy and had not been exposed to chorioamnionitis in utero. Expression of these activation markers is independent of phagocytosis, suggesting the altered phagocytic capability of preterm and term infants is not due to activation state of their phagocytic cells.

The role of complement in phagocytosis is critical, opsonizing bacteria to aid engulfment and removal of infection (14). Newborns, particularly preterm infants, have decreased levels and function of complement pathways $(9,10,40)$. Phagocytosis of GBS, SE, and EC by isolated phagocytes has been shown to increase with, or be complement-dependent $(25,41,42)$. However, in the presence of native complement in whole blood, we have shown that complement from preterm infants is not deficient or functionally defective for the uptake of SE and SA. We have also previously reported similar observations for GBS phagocytosis (24). The important observation that restoration of the proportion of preterm infant cells capable of phagocytosing EC to term infant levels with the addition of rabbit complement suggests that EC phagocytosis is indeed complement-dependent. Of note, neutrophils appear to be more sensitive to complement than monocytes for phagocytosis of EC, with an increase in both term and preterm infant phagocytic cells observed with the addition of exogenous complement. Our findings indicate that a complement deficiency or malfunction in preterm infants is not responsible for the differences in phagocytosis of SE or SA between preterm and term infants, but does contribute to decreased phagocytosis of EC.
In conclusion, we have identified a decrease in number but an increase in functionality of phagocytic cells in preterm compared with term infant whole blood. These differences and the magnitude of phagocytosis measured are comparable in cord and $<24 \mathrm{~h}$ peripheral blood, confirming cord as a reasonable surrogate for peripheral samples immediately after birth. Deficiencies in phagocyte activation in preterm infants were not responsible for the disparities between term and preterm phagocytosis; however, complement plays a role in the proportion of cells capable of ingesting EC. Preferential phagocytosis of SE over SA and EC suggest pathogen-specific phagocytosis functionality. The immune defects underlying the increased susceptibility of preterm infants to SE may therefore reside in facets of the innate immune response other than phagocytosis. These findings warrant additional investigation, particularly at later time-points to identify further differences in the development of phagocytosis functionality between preterm and term infants, and whether these contribute to the heightened susceptibility to infection in preterm infants.

\section{METHODS}

\section{Blood Sampling and Preparation}

The King Edward Memorial Hospital (Perth, Australia) Ethics Committee approved the study protocol and written, informed consent was obtained $(814 / \mathrm{EW})$. Cord blood was collected into preheparinized syringes as soon as possible (within $4 \mathrm{~h}$ ) postnatally from cord vessels, and where necessary, from vessels feeding into cord vessels, immediately adjacent to the base of the cord on the fetal placental surface. Collection sites were alcohol swabbed before sampling to minimize possible maternal blood contamination. The majority of preterm cord samples ( 20 out of 26 ) were collected $<1 \mathrm{~h}$ after delivery (median collection time $14 \mathrm{~min}$ ), four were collected between 1 and $2 \mathrm{~h}$ after delivery (median collection time of $100 \mathrm{~min}$ ) and only two were collected $3 \mathrm{~h}$ postdelivery. All term infant cord samples were collected $<2 \mathrm{~h}$ after delivery (median collection time of $74 \mathrm{~min}$ ). Infant $<24 \mathrm{~h}$ peripheral venous blood samples were collected into heparinized tubes within $24 \mathrm{~h}$ of birth. To exclude possible confounding by concurrent infection, we excluded infants with histological evidence of chorioamnionitis or with subsequent early-onset sepsis. The demographics of each donor group were: 58 preterm infants (27 female) median 27.6 wks GA (range: 23.4-30.1) and 20 term infants (11 female) median 40 wks GA (range: 37.4-42).

Heparinized blood samples from healthy adults were used to optimize experimental conditions and minimize the blood volume for subsequent infant samples. Additionally, heparinized adult blood samples were left for up to $48 \mathrm{~h}$ at room temperature (RT) after collection, before the phagocytosis assay, to determine the reliability of neutrophil and monocyte phagocytosis when measurement was delayed. The stability of the pHrodo signal after phagocytosis in monocytes and neutrophils was determined by storing adult samples, postassay, at $4{ }^{\circ} \mathrm{C}$ in the dark for 1 or $3 \mathrm{~d}$ before flow cytometry to determine the reliability of batch analysis. No significant differences in fluorescence intensities were observed between batches (data not shown).

\section{pHrodo Labeling of Bacteria}

The SE isolate (wild-type strain 1457) was originally from a patient with an infected central venous catheter. Stocks were grown to mid$\log$ phase $\left(\mathrm{OD}_{600}: 0.7-0.8\right)$ in Heart Infusion Broth (Oxoid, Adelaide, Australia) and heat-killed at $80^{\circ} \mathrm{C}$ for $15 \mathrm{~min}$. Postkilling nonviability of the SE was confirmed by culture. Total bacterial counts were made using a Helber Bacteria Counting Chamber (ProSciTech, Thuringowa, Australia). Bacteria were washed in pyrogen-free phosphate-buffered saline (PBS) and counted before labeling with pHrodo dye (Invitrogen, Mulgrave, Australia), in keeping with the manufacturer's protocol. Briefly, $2.5 \times 10^{9}$ bacterial cells were equilibrated to $\mathrm{pH} 8.5$ by washing 
in $100 \mathrm{mmol} / \mathrm{l}$ sodium bicarbonate. Cells were resuspended in $750 \mu \mathrm{l}$ sodium bicarbonate and incubated with $0.5 \mathrm{mmol} / \mathrm{l}$ pHrodo dye for $45 \mathrm{~min}$ at RT. Several wash steps with methanol and PBS removed excess dye. Labeled bacteria were resuspended in $\mathrm{H}_{2} \mathrm{O}$ and either used immediately or lyophilized and frozen $\left(-20^{\circ} \mathrm{C}\right.$ for future reconstitution). Bacteria were vortexed and sonicated to ensure single cell suspensions before counting. The $\mathrm{pH}$ response range of the dye was routinely verified by comparing fluorescence in PBS of pH 7.4 and pH 4.0.

Commercial stocks of prelabeled, lyophilized SA and EC strain K12 (both from Invitrogen) were reconstituted in $\mathrm{H}_{2} \mathrm{O}$ before counting and aliquots were lyophilized and stored at $-20^{\circ} \mathrm{C}$. SA, EC, and SE stocks were stored at a concentration of $2.5 \times 10^{9} / \mathrm{ml}$.

\section{Phagocytosis Assay}

Whole blood $(25 \mu \mathrm{l})$ was transferred to wells of polypropylene 96-well round-bottomed plates (Corning Inc., Corning, NY) with complete medium (RPMI, Gibco, Life Technology, Paisley, Scotland) with 5\% heat-inactivated fetal calf serum (MultiSer Biosciences, Castle Hill, Australia), $10 \mathrm{mmol} / \mathrm{l} \mathrm{HEPES,} 1 \mathrm{mmol} / \mathrm{l}$ sodium pyruvate and 550 $\mu \mathrm{mol} / \mathrm{l} \beta$-mercaptoethanol (all Invitrogen) to a final volume of $120 \mu \mathrm{l}$. Samples were incubated either with or without $10 \%(\mathrm{v} / \mathrm{v})$ baby RbC PelFreez, Rogers, AR).

Samples were incubated at $37^{\circ} \mathrm{C}$ with EC, SA, or SE at $10^{8}$ bacteria/ $\mathrm{ml}$ for $15 \mathrm{~min}$, before transfer to ice to halt phagocytosis. Cytochalasin D $(20 \mu \mathrm{g} / \mathrm{ml}$; Sigma-Aldrich, Sydney, Australia) was added to some experiments using adult blood, $10 \mathrm{~min}$ before addition of bacteria to inhibit phagocytosis. The multiplicity of infection value was $~ 50: 1$ (bacteria:phagocyte) based on the median of accepted ranges of phagocytes (total neutrophils plus monocytes) in neonates $\left(12 \times 10^{9} / 1(43)\right)$. Samples were then incubated with $1 \mu \mathrm{l}$ of antihuman CD14-fluorescein isothiocyanate (FITC) (BD, North Ryde, Australia) on ice for $15 \mathrm{~min}$. Cells were washed twice with $2 \mathrm{ml}$ ice-cold PBS and red blood cells were lysed by incubation with $1 \mathrm{ml}$ FACSLyse (BD) for $15 \mathrm{~min}$ at RT. Cells were washed once in PBS, resuspended in Stabilizing Fixative (BD) and stored at $4{ }^{\circ} \mathrm{C}$ in the dark before analysis. Monocytes were subsequently identified during FACS analysis as SSC ${ }^{\text {int }} \mathrm{CD} 14^{+}$cells, and neutrophils as $\mathrm{SSC}^{\text {hi }} \mathrm{CD} 14^{-}$cells. Validation checks using adult blood samples demonstrated the pHrodo signal in fixed neutrophils and monocytes was stable for at least $3 \mathrm{~d}$ at $4{ }^{\circ} \mathrm{C}$ (data not shown), supporting batch acquisition of sample data by FACS.

\section{Microscopy Confirming Phagocytosis}

Adult mononuclear cells were coincubated with an multiplicity of infection of 100:1 pHrodo-labeled SE for $1 \mathrm{~h}$ at $37^{\circ} \mathrm{C}$ with or without pretreatment with $20 \mu \mathrm{g} / \mathrm{ml} \mathrm{CD}$ for $10 \mathrm{~min}$. Cells were harvested, washed in PBS and cyto-centrifuged onto microscopy slides. Nuclear staining was performed using Hoechst 33342 (Invitrogen) and mounted using low-fade mounting media. Fluorescent and transmission imaging used a Bio-Rad MRC 1000 confocal laser-scanning microscope (Bio-Rad Laboratories Pty., Ltd., Gladesville, Australia) with PlanApo objectives.

\section{Activation Marker Expression Analysis}

Separate aliquots of blood $(150 \mu \mathrm{l})$ were incubated with the following antibodies; Lin-2 FITC and HLA-DR PECy7 together with either CD86 APC and CD64 PE or matched isotype controls (all from BD) for $15 \mathrm{~min}$ at RT. Cells were lysed with $2 \mathrm{ml}$ FACSLyse (BD) for $15 \mathrm{~min}$ at RT, then washed and resuspended in stabilizing fixative (BD) and stored at $4{ }^{\circ} \mathrm{C}$ in the dark before analysis.

\section{Flow Cytometry}

Assay optimization experiments were analyzed using a BD FACSCalibur dual-laser flow cytometer (BD) and validation experiments were acquired with a BD FACSCanto II triple-laser machine (BD). Twocolor analysis of green and red fluorescence detected CD14-FITC positive monocytes and pHrodo positive phagocytes, respectively. Detection of FITC fluorescence was achieved on both flow cytometers using a 530/30 nm band-pass filter while pHrodo fluorescence was detected through a $670 \mathrm{~nm}$ long-pass filter. No compensation for the two parameters was required. Equivalent fluorescence intensities for both machines were established using SPHERO Rainbow calibration beads (SpheroTech Inc., Lake Forest, IL) and checked weekly. Untreated whole blood samples served to determine the cut-off for fluorescence for phagocytosis and 2,000 monocytes were collected from each sample.

Activation marker assessment experiments were analyzed on the same FACSCanto II flow cytometer using the following bandpass filters for detection; FITC: 530/30 nm, PECy7: $780 / 60 \mathrm{~nm}$, APC: $660 / 20 \mathrm{~nm}$, and PE: $585 / 42 \mathrm{~nm}$. Compensation controls were included for each fluorophore and the matrix was calculated and applied using Flowjo software (Tree Star, Ashland, OR). Monocytes $(>2,000)$ were identified as Lin- $2^{+} \mathrm{HLA}-\mathrm{DR}^{+}$and neutrophils as Lin$2^{-}$SSC $^{\text {hi }}$. Matched isotype controls for CD64 and CD86 were included to determine the gate identifying positive expression. All data were analyzed with Flowjo software.

\section{Statistical Analysis}

Two-group comparisons were made by Mann-Whitney $U$ and Spearman's test was used for analysis of correlations. Analyses were conducted using Prism 5 for Mac (GraphPad, La Jolla, CA). Data presented as bar graphs are shown as mean \pm SEM. Differences were considered significant at $P<0.05$.

\section{ACKNOWLEDGMENTS}

The authors thank Gail Abernethy and Annie Chang for excellent assistance with recruitment and sample collection. The 1457 wild-type isolate of SE used in the study was kindly provided by Michael Otto (National Institute of Allergy and Infectious Diseases, Bethesda, MD). The authors thank the Princess Margaret Hospital for Children Department of Immunology for provision of flow cytometry equipment and assistance. Technical assistance from Ruth Thornton is gratefully acknowledged. The authors also thank all participating families and donors.

\section{STATEMENT OF FINANCIAL SUPPORT}

This study was supported by Australian National Health and Medical Research Council project grants 513847 and 572548. Andrew Currie is supported by a fellowship from the BrightSpark Foundation of Western Australia. David Burgner is supported by a National Health and Medical Research Council Career Development Fellowship and the Victorian Government's Operational Infrastructure Support Program.

Disclosure: The authors have no conflicts of interest to declare.

\section{REFERENCES}

1. Jutras I, Desjardins M. Phagocytosis: at the crossroads of innate and adaptive immunity. Annu Rev Cell Dev Biol 2005;21:511-27.

2. Blundell MP, Worth A, Bouma G, Thrasher AJ. The Wiskott-Aldrich syndrome: The actin cytoskeleton and immune cell function. Dis Markers 2010;29:157-75.

3. Isaacs D; Australasian Study Group For Neonatal Infections. A ten year, multicentre study of coagulase negative staphylococcal infections in Australasian neonatal units. Arch Dis Child Fetal Neonatal Ed 2003;88:F89-93.

4. Stoll BJ, Gordon T, Korones SB, et al. Early-onset sepsis in very low birth weight neonates: a report from the National Institute of Child Health and Human Development Neonatal Research Network. J Pediatr 1996;129:72-80.

5. Sgro M, Shah PS, Campbell D, Tenuta A, Shivananda S, Lee SK; Canadian Neonatal Network. Early-onset neonatal sepsis: rate and organism pattern between 2003 and 2008. J Perinatol 2011;31:794-8.

6. Stoll BJ, Hansen N, Fanaroff AA, et al. Changes in pathogens causing earlyonset sepsis in very-low-birth-weight infants. N Engl J Med 2002;347:2407.

7. Johri AK, Paoletti LC, Glaser P, et al. Group B Streptococcus: global incidence and vaccine development. Nat Rev Microbiol 2006;4:932-42.

8. Cohen-Wolkowiez M, Moran C, Benjamin DK, et al. Early and late onset sepsis in late preterm infants. Pediatr Infect Dis J 2009;28:1052-6.

9. Levy O. Innate immunity of the newborn: basic mechanisms and clinical correlates. Nat Rev Immunol 2007;7:379-90.

10. Strunk T, Currie A, Richmond P, Simmer K, Burgner D. Innate immunity in human newborn infants: prematurity means more than immaturity. J Matern Fetal Neonatal Med 2011;24:25-31.

11. Gille C, Spring B, Tewes L, Poets CF, Orlikowsky T. A new method to quantify phagocytosis and intracellular degradation using green fluorescent 
protein-labeled Escherichia coli: comparison of cord blood macrophages and peripheral blood macrophages of healthy adults. Cytometry A 2006;69:152-4.

12. Hallwirth U, Pomberger G, Pollak A, Roth E, Spittler A. Monocyte switch in neonates: high phagocytic capacity and low HLA-DR expression in VLBWI are inverted during gestational aging. Pediatr Allergy Immunol 2004;15:513-6.

13. Maródi L, Leijh PC, van Furth R. Characteristics and functional capacities of human cord blood granulocytes and monocytes. Pediatr Res 1984;18:1127-31.

14. Schutze GE, Hall MA, Baker CJ, Edwards MS. Role of neutrophil receptors in opsonophagocytosis of coagulase-negative staphylococci. Infect Immun 1991;59:2573-8.

15. Gille Ch, Leiber A, Mundle I, et al. Phagocytosis and postphagocytic reaction of cord blood and adult blood monocyte after infection with green fluorescent protein-labeled Escherichia coli and group B Streptococci. Cytometry B Clin Cytom 2009;76:271-84.

16. Strunk T, Temming P, Gembruch U, Reiss I, Bucsky P, Schultz C. Differential maturation of the innate immune response in human fetuses. Pediatr Res 2004;56:219-26.

17. Howie SR. Blood sample volumes in child health research: review of safe limits. Bull World Health Organ 2011;89:46-53.

18. Bernardo J, Long HJ, Simons ER. Initial cytoplasmic and phagosomal consequences of human neutrophil exposure to Staphylococcus epidermidis. Cytometry A 2010;77:243-52.

19. Miksa M, Komura H, Wu R, Shah KG, Wang P. A novel method to determine the engulfment of apoptotic cells by macrophages using pHrodo succinimidyl ester. J Immunol Methods 2009;342:71-7.

20. Notarangelo LD, Chirico G, Chiara A, et al. Activity of classical and alternative pathways of complement in preterm and small for gestational age infants. Pediatr Res 1984;18:281-5.

21. Fabbrini M, Sammicheli C, Margarit I, et al. A new flow-cytometrybased opsonophagocytosis assay for the rapid measurement of functional antibody levels against Group B Streptococcus. J Immunol Methods 2012;378:11-9.

22. Falconer AE, Carr R, Edwards SW. Impaired neutrophil phagocytosis in preterm neonates: lack of correlation with expression of immunoglobulin or complement receptors. Biol Neonate 1995;68:264-9.

23. Fujiwara T, Kobayashi T, Takaya J, Taniuchi S, Kobayashi Y. Plasma effects on phagocytic activity and hydrogen peroxide production by polymorphonuclear leukocytes in neonates. Clin Immunol Immunopathol 1997;85: 67-72.

24. Currie AJ, Curtis S, Strunk T, et al. Preterm infants have deficient monocyte and lymphocyte cytokine responses to group B streptococcus. Infect Immun 2011;79:1588-96.

25. Strunk T, Prosser A, Levy O, et al. Responsiveness of human monocytes to the commensal bacterium Staphylococcus epidermidis develops late in gestation. Pediatr Res 2012;72:10-8.

26. Härtel C, Osthues I, Rupp J, et al. Characterisation of the host inflammatory response to Staphylococcus epidermidis in neonatal whole blood. Arch Dis Child Fetal Neonatal Ed 2008;93:F140-5.
27. Sadeghi K, Berger A, Langgartner M, et al. Immaturity of infection control in preterm and term newborns is associated with impaired toll-like receptor signaling. J Infect Dis 2007;195:296-302.

28. Hallwirth U, Pomberger G, Zaknun D, et al. Monocyte phagocytosis as a reliable parameter for predicting early-onset sepsis in very low birthweight infants. Early Hum Dev 2002;67:1-9.

29. Eder K, Vizler C, Kusz E, et al. The role of lipopolysaccharide moieties in macrophage response to Escherichia coli. Biochem Biophys Res Commun 2009;389:46-51.

30. Katzenmeyer KN, Bryers JD. Multivalent artificial opsonin for the recognition and phagocytosis of Gram-positive bacteria by human phagocytes. Biomaterials 2011;32:4042-51.

31. Peterson PK, Wilkinson BJ, Kim Y, Schmeling D, Quie PG. Influence of encapsulation on staphylococcal opsonization and phagocytosis by human polymorphonuclear leukocytes. Infect Immun 1978;19: 943-9.

32. Gordon DL, Avery VM, Rice JL, McDonald PJ. Surface phagocytosis of Staphylococcus epidermidis and Escherichia coli by human neutrophils: serum requirements for opsonization and chemiluminescence. FEMS Microbiol Immunol 1989;1:417-23.

33. Strunk T, Richmond P, Prosser A, et al. Method of bacterial killing differentially affects the human innate immune response to Staphylococcus epidermidis. Innate Immun 2011;17:508-16.

34. Manjuck J, Saha DC, Astiz M, Eales LJ, Rackow EC. Decreased response to recall antigens is associated with depressed costimulatory receptor expression in septic critically ill patients. J Lab Clin Med 2000;135:153-60.

35. Fjaertoft G, Håkansson L, Ewald U, Foucard T, Venge P. Neutrophils from term and preterm newborn infants express the high affinity Fcgamma-receptor I (CD64) during bacterial infections. Pediatr Res 1999;45:871-6.

36. Birle A, Nebe CT, Gessler P. Age-related low expression of HLA-DR molecules on monocytes of term and preterm newborns with and without signs of infection. J Perinatol 2003;23:294-9.

37. Pérez A, Bellón JM, Gurbindo MD, Muñoz-Fernández MA. Impairment of stimulation ability of very-preterm neonatal monocytes in response to lipopolysaccharide. Hum Immunol 2010;71:151-7.

38. Kanakoudi-Tsakalidou F, Debonera F, Drossou-Agakidou V, et al. Flow cytometric measurement of HLA-DR expression on circulating monocytes in healthy and sick neonates using monocyte negative selection. Clin Exp Immunol 2001;123:402-7.

39. Hoffmann JJ. Neutrophil CD64 as a sepsis biomarker. Biochem Med (Zagreb) 2011;21:282-90.

40. Fleer A, Gerards LJ, Aerts P, et al. Opsonic defense to Staphylococcus epidermidis in the premature neonate. J Infect Dis 1985;152:930-7.

41. Clark LA, Easmon CS. Opsonic requirements of Staphylococcus epidermidis. J Med Microbiol 1986;22:1-7.

42. Bektas S, Goetze B, Speer CP. Decreased adherence, chemotaxis and phagocytic activities of neutrophils from preterm neonates. Acta Paediatr Scand 1990;79:1031-8.

43. McClatchey K. Clinical Laboratory Medicine, 2nd ed. Philadelphia, PA: Lippincott Williams \& Wilkins, 2002, 807. 